\title{
Herpes Simplex Virus
}

National Cancer Institute

\section{Source}

National Cancer Institute. Herpes Simplex Virus. NCI Thesaurus. Code C112419.

A genus of enveloped, spherical or pleomorphic viruses with a capsid with $T=16$

icosahedral symmetry and surrounded by amorphous tegument, in the family

Herpesviridae. The genome is composed of monopartite, linear double-stranded DNA.

Simplexviruses are found in humans and mammals, may remain latent in neurons and cause skin blisters and mucosal ulcers in humans. 